\title{
ON CERTAIN RELATIONS BETWEEN THE PROJECTIVE THEORY OF SURFACES AND THE PROJECTIVE THEORY OF CONGRUENCES*
}

\author{
BY \\ FRANK EDWIN WOOD
}

1. INTRODUCTION

Wilczynski has shown $\dagger$ that the system of partial differential equations

$$
\begin{aligned}
y_{v} & =m z, \quad z_{u}=n y \\
y_{u u} & =a y+b z+c y_{u}+d z_{v} \\
z_{v v} & =a^{\prime} y+b^{\prime} z+c^{\prime} y_{u}+d^{\prime} z_{v}
\end{aligned}
$$

where

$$
y_{u}=\frac{\partial y}{\partial u}, \quad y_{v}=\frac{\partial y}{\partial v}, \quad y_{u u}=\frac{\partial^{2} y}{\partial u^{2}}, \text { etc. }
$$

and where $m, n, a, \ldots, d^{\prime}$ are functions of $u$ and $v$, will be completely integrable if the following integrability conditions are satisfied:

$$
\begin{aligned}
& c=f_{u}, d^{\prime}=f_{v}, b=-d_{v}-d f_{v}, a^{\prime}=-c^{\prime}{ }_{u}-c^{\prime} f_{u}, m n-c^{\prime} d=f_{u v}, \\
& m_{u u}+d_{v v}+d f_{v v}+d_{v} f_{v}-f_{u} m_{u}=m a+d b^{\prime}, \\
& n_{v v}+c^{\prime}{ }_{u u}+c^{\prime} f_{u u}+c^{\prime}{ }_{u} f_{u}-f_{v} n_{\imath}=c^{\prime} a+n b^{\prime}, \\
& 2 m_{u} n+m n_{u}=a_{v}+f_{u} m n+a^{\prime} d, \\
& m_{v} n+2 m n_{v}=b^{\prime}{ }_{u}+f_{v} m n+b c^{\prime} .
\end{aligned}
$$

In such a case, (1) will have precisely four linearly independent solutions $\left(y^{(K)}, z^{(K)}\right)(K=1,2,3,4)$. Let $y^{(1)}, \ldots, y^{(4)}$ and $z^{(1)}, \ldots, z^{(4)}$ be interpreted as the homogeneous coördinates of the two points $P_{y}$ and $P_{z}$. As $u$ and $v$ vary, these points will describe two surfaces $S_{y}$ and $S_{z}$ and the line $P_{y} P_{z}$ will generate a congruence whose focal surface consists of the two surfaces $S_{y}$ and $S_{z}$.

* Presented to the Society, under a different title, March 26, 1921.

† E. J. Wilczynski, Sur la théorie générale des congruences, M é m o i r e s $\mathrm{P}$ u b 1 i é $\mathrm{s}$ par la Classedes Sciences de 1'Académie Royalde Belgique, Colle c $\mathrm{t}$ i o n e $4,^{\circ}$ ser. 2, vol. 3 (1911). This paper will hereafter be cited as the Brussels Paper. 
The most general transformation which will leave the set (1) in the same form is

$$
y=\lambda(u) \bar{y}, \quad z=\mu(v) \bar{z}, \quad \bar{u}=\alpha(u), \quad \bar{v}=\beta(v)
$$

where $\lambda, \mu, \alpha, \beta$, are arbitrary functions of the variables indicated. The coefficients $\bar{m}, \bar{n}, \bar{a}, \ldots, \bar{d}^{\prime}$ resulting from this transformation are

$$
\begin{aligned}
\bar{m} & =\frac{\mu}{\lambda \beta_{v}} m, \quad \bar{n}=\frac{\lambda}{\mu \alpha_{u}} n, \bar{d}=\frac{\mu \beta_{v}}{\lambda \alpha_{u}^{2}} d, \bar{c}^{\prime}=\frac{\lambda \alpha_{u}}{\mu \beta_{v}^{2}} c^{\prime}, \\
\text { (4) } \bar{a} & =\frac{1}{\alpha_{u}^{2}}\left(a+\frac{\lambda_{u}}{\lambda} c-\frac{\lambda_{u u}}{\lambda}\right), \bar{b}=\frac{\mu}{\lambda \alpha_{u}^{2}}\left(b+\frac{\mu_{v}}{\mu} d\right), \bar{c}=\frac{1}{\alpha_{u}}\left(c-2 \frac{\lambda_{u}}{\lambda}-\frac{\alpha_{u u}}{\alpha_{u}}\right), \\
\bar{a}^{\prime} & =\frac{\lambda}{\mu \beta_{v}^{2}}\left(a^{\prime}+\frac{\lambda_{u}}{\lambda} c^{\prime}\right), \bar{b}^{\prime}=\frac{1}{\beta_{v}^{2}}\left(b^{\prime}+\frac{\mu_{v}}{\mu} d^{\prime}-\frac{\mu_{v v}}{\mu}\right), \bar{d}^{\prime}=\frac{1}{\beta_{v}}\left(d^{\prime}-2 \frac{\mu_{v}}{\mu}-\frac{\beta_{v v}}{\beta_{v}}\right) ;
\end{aligned}
$$

to which may also be added

$$
\overline{(e)}=\frac{K e^{f}}{\lambda^{2} \mu^{2} \alpha_{u} \beta_{v}}
$$

where $K$ is an arbitrary constant.

There are various covariant configurations connected with the congruence. Some of these are connected with the focal surface and present themselves at first referred to a local coördinate system connected with that surface. Others appear directly in their relation to the local coördinate system of the congruence. In order that we may study the relations between these covariant configurations, their equations must be related to a common tetrahedron of reference. The fundamental covariants of the congruence form a tetrahedron, and the relations which exist between this tetrahedron and the local tetrahedra of reference for $S_{y}$ and $S_{z}$, respectively, must therefore be obtained. The development of these relations constitutes the first part of this article.

There are two asymptotic curves which pass through a point $P_{y}$ on $S_{y}$, and the osculating linear complexes of these two curves have a linear congruence in common. The two directrices of this congruence are called* $\left(d_{1}{ }^{\prime}\right)$ the directrix of $P_{y}$ of the first kind; this directrix lies in the tangent plane of $S_{y}$ at $P_{y}$ but does not contain $P_{y}$, and $\left(d_{1}{ }^{\prime \prime}\right)$ the directrix of $P_{y}$ of the second kind; this directrix. passes through $P_{y}$ but does not lie in the tangent plane.

The curves cut out on $S_{y}$ by the parametric curves of the congruence form a conjugate system; the osculating planes at $P_{y}$ of these two curves intersect in

* E. J. Wilczynski, Projective differential geometry of curved surfaces (Second Memoir), these T r a n s a c t i o n s, vol. 9 (1908), p. 95. 
a line which passes through $P_{y}$ and is called* $\left(x_{1}\right)$ the axis of the point $P_{y}$ with respect to the conjugate system.

The dual of the axis of $P_{y}$ is called $\dagger\left(r_{1}\right)$ the ray of $P_{y}$ with respect to the conjugate system.

There are four lines $d_{2}^{\prime}, d_{2}^{\prime \prime}, x_{2}, r_{2}$ similarly connected with $P_{z}$ on $S_{z}$ making eight lines in all. In the second part of this paper, the equations of these eight lines referred to the tetrahedron of the congruence are obtained.

In the remaining parts of this article the possible coincidences of two or more of these lines are considered in general, and congruences which are characterized by certain pairs of coincidences are obtained in a canonical form. The existence of congruences which have no one of the eight associated lines indeterminate and which possess any one of twelve possible coincidences is established. In particular a study is made of the congruences which possess one of the following coincidence pairs: (1) the directrix of the first kind and the ray of $P_{y}$ coincide, and the directrix of the second kind of $P_{y}$ coincides with the ray of $P_{z}$, or (2) the axis of $P_{y}$ coincides with the ray of $P_{z}$, and the ray of $P_{y}$ coincides with the axis of $P_{z}$, or (3) the directrix of the first kind of $P_{y}$ coincides with the directrix of the second kind of $P_{z}$, and the directrix of the second kind of $P_{y}$ coincides with the directrix of the first kind of $P_{z}$. This class of congruence has a special interest since it can be connected with the theory of functions of a complex variable.

The writer wishes to thank Professor Wilczynski for his kindly interest and valuable suggestions.

2. RELATIONS BETWEEN THE TETRAHEDRA OF REFERENCE OF THE FOCAL SHEET AND THE TETRAHEDRON OF THE CONGRUENCE

The differential equations which characterize $S_{y}$ are

$$
\begin{aligned}
y_{u u}-\frac{d}{m} y_{v v} & =a y+c y_{u}+\left(\frac{b}{m}-\frac{d}{m} \frac{m_{v}}{m}\right) y_{v}, \\
y_{u v} & =m n y+\frac{m_{u}}{m} y_{v} .
\end{aligned}
$$

Make the transformation

$$
u_{1}=\varphi(u, v), \quad v_{1}=\psi(u, v)
$$

* E. J. Wilczynski, The general theory of congruences, these $\mathrm{T}$ r a n s a c t i o n s, vol. 16 (1915), p. 314.

$\dagger$ Loc. cit., pp. 317-18.

$\ddagger$ E. J. Wilczynski, Brussels Paper, p. 44, equation 79. 
where

$$
\varphi_{u}=\delta \varphi_{v}, \quad \psi_{u}=-\delta \psi_{v}, \quad \delta^{2}=-\frac{d}{m}
$$

The new parametric curves $u_{1}=$ const. and $v_{1}=$ const. will be the asymptotic curves of $S_{y}$ and the transform of (5) will be in the intermediate form.*

Let $F(u, v)$ be a general function; then

$$
\begin{aligned}
& F_{u}=\frac{\partial F}{\partial u}=\frac{\partial F}{\partial u} \frac{\partial \varphi}{\partial u}+\frac{\partial F}{\partial v_{1}} \frac{\partial \psi}{\partial u}=\delta\left(\varphi_{v} F_{u_{1}}-\psi_{v} F_{v_{1}}\right), \\
& F_{v}=\frac{\partial F}{\partial v}=\frac{\partial F}{\partial u_{1}} \frac{\partial \varphi}{\partial v}+\frac{\partial F}{\partial v_{1}} \frac{\partial \psi}{\partial v}=\varphi_{v} F_{u_{1}}+\psi_{v} F_{v_{1}}
\end{aligned}
$$

whence

$$
\begin{aligned}
& F_{u_{1}}=\frac{1}{2 \delta \varphi_{v}}\left(F_{u}+\delta F_{v}\right), \\
& F_{v_{1}}=\frac{1}{2 \delta \psi_{v}}\left(-F_{u}+\delta F_{v}\right) .
\end{aligned}
$$

Using (6) and (7) one obtains

$$
\begin{aligned}
& y_{u}=\delta\left(\varphi_{v} y_{u_{1}}-\psi_{v} y_{v_{1}}\right), \quad y_{v}=\varphi_{v} y_{u_{1}}+\psi_{v} y_{v_{1}} \\
& y_{u u}=\delta^{2}\left(\varphi_{v}^{2} y_{u_{1} u_{1}}-2 \varphi_{v} \psi_{v} y_{u_{1} v_{1}}+\psi_{v}^{2} y_{v_{1} v_{1}}\right)+\left(\delta \varphi_{u v}+\delta_{u} \varphi_{v}\right) y_{u_{1}}
\end{aligned}
$$

$y_{u v}=\delta\left(\varphi_{v}^{2} y_{u_{1} u_{1}}-\psi_{v}^{2} y_{v_{1} v_{1}}\right)+\left(\delta \varphi_{v v}+\delta_{v} \varphi_{v}\right) y_{u_{1}}-\left(\delta \psi_{v v}+\delta_{v} \psi_{v}\right) y_{v_{1}}$,

$y_{v v}=\varphi_{v}^{2} y_{u_{1} u_{1}}+2 \varphi_{v} \psi_{v} y_{u_{1} v_{1}}+\psi_{v}^{2} y_{v_{1} v_{1}}+\varphi_{v v} y_{u_{1}}+\psi_{v v} y_{v_{1}}$.

If these values (8) be substituted in (5), we find the differential equations of $S_{y}$ referred to its asymptotic lines. These equations are

$$
\begin{aligned}
& y_{u_{1} u_{1}}+2 a_{1} y_{u_{1}}+2 b_{1} y_{v_{1}}+c_{1} y=0, \\
& y_{v_{1} v_{1}}+2 a_{1}^{\prime} y_{u_{1}}+2 b_{1}^{\prime} y_{v_{1}}+c_{1}^{\prime} y=0,
\end{aligned}
$$

where

$$
\begin{aligned}
& 2 a_{1}=\frac{1}{4 \delta^{2} \varphi_{v}}\left(4 \delta^{2} \frac{\varphi_{v v}}{\varphi_{v}}-\delta c-\frac{b}{m}-\delta^{2} \frac{m_{v}}{m}-2 \delta \frac{m_{u}}{m}+3 \delta \delta_{v}+\delta_{u}\right), \\
& 2 b_{1}=\frac{\psi_{v}}{4 \delta^{2} \varphi_{v}^{2}}\left(-\delta c-\frac{b}{m}-\delta^{2} \frac{m_{v}}{m}-2 \delta \frac{m_{u}}{m}-\delta \delta_{v}-\delta_{u}\right), \\
& c_{1}=-\frac{1}{4 \delta^{2} \varphi_{v}^{2}}(a+2 \delta m n), \\
& 2 a_{1}^{\prime}=\frac{\varphi_{v}}{4 \delta^{2} \psi_{v}^{2}}\left(-\delta c-\frac{b}{m}-\delta^{2} \frac{m_{v}}{m}+2 \delta \frac{m_{u}}{m}-\delta \delta_{v}+\delta_{u}\right), \\
& 2 b_{1}^{\prime}=\frac{1}{4 \delta^{2} \psi_{v}}\left(4 \delta^{2} \frac{\psi_{v v}}{\psi_{v}}+\delta c-\frac{b}{m}-\delta^{2} \frac{m_{v}}{m}+2 \delta \frac{m_{u}}{m}+3 \delta \delta_{v}-\delta_{u}\right), \\
& c_{1}^{\prime}=-\frac{1}{4 \delta^{2} \psi_{v}^{2}}(a-2 \delta m n) .
\end{aligned}
$$

* E. J. Wilczynski, Projective differential geometry of curved surfaces (Second Memoir), these Transactions, vol. 9 (1908). 
Let us introduce the notations*

$$
\begin{aligned}
& B_{1}=\frac{1}{4}\left(c-2 \frac{m_{u}}{m}-\frac{\delta_{u}}{\delta}\right)=\frac{1}{4} \frac{\partial}{\partial u}\left(f-\log \delta m^{2}\right)=\frac{1}{4}\left(f_{u}-\frac{1}{2} \frac{d_{u}}{d}-\frac{3}{2} \frac{m_{u}}{m}\right) \\
& C_{1}=-\frac{1}{4}\left(\frac{b}{d}-\frac{m_{v}}{m}-\frac{\delta_{v}}{\delta}\right)=\frac{1}{4} \frac{\partial}{\partial v}\left(f+\log \delta^{3} m^{2}\right)=\frac{1}{4}\left(f_{v}+\frac{3}{2} \frac{d_{v}}{d}+\frac{1}{2} \frac{m_{v}}{m}\right) \\
& F_{1}=-\frac{1}{\delta} B_{1}-C_{1} \\
& G_{1}=\frac{1}{\delta} B_{1}-C_{1}
\end{aligned}
$$

then equations (10) become

$$
\begin{aligned}
& 2 a_{1}=\frac{1}{\varphi_{v}}\left(\frac{\varphi_{v v}}{\varphi_{v}}+F_{1}-\frac{1}{\delta} \frac{m_{u}}{m}+\frac{\delta_{v}}{\delta}\right), 2 a_{1}^{\prime}=\frac{\varphi}{\psi_{v}^{2}} F_{1}, \\
& 2 b_{1}=\frac{\psi_{v}}{\varphi_{v}^{2}} G_{1}, 2 b_{1}^{\prime}=\frac{1}{\psi_{v}}\left(\frac{\psi_{2 v}}{\psi_{v}}+F_{1}+\frac{1}{\delta} \frac{m_{u}}{m}+\frac{\delta_{v}^{s}}{\delta}\right), \\
& c_{1}=-\frac{1}{4 \delta^{2} \varphi_{v}^{2}}(a+2 \delta m n), c_{1}^{\prime}=-\frac{1}{4 \delta^{2} \psi_{v}^{2}}(a-2 \delta m n) .
\end{aligned}
$$

The fundamental semicovariants of $S_{y}$ are $\dagger$

$$
\begin{aligned}
& y_{1}=y, \quad z_{1}=y_{u_{1}}+a_{1} y, \quad \rho_{1}=y_{v_{1}}+b_{1}^{\prime} y, \\
& \sigma_{1}=y_{u_{1} v_{1}}+b_{1}^{\prime} y_{u_{1}}+a_{1} y_{v_{1}}^{\prime}+\frac{1}{2}\left[\left(a_{1}\right)_{v_{1}}+\left(b_{1}^{\prime}\right)_{u_{1}}+2 a_{1} b_{1}^{\prime}\right] y,
\end{aligned}
$$

and the fundamental relative covariants of the congruence are $\ddagger$

$$
y=y, z=z, \rho \doteq y_{u}-\frac{m_{u}}{m} y, \sigma=z_{v}-\frac{n_{v}}{n} z:
$$

From (1), (7), (13) and (14) the following relations are obtained:

$$
\begin{aligned}
& y_{1}=y, \\
& z_{1}=\left(a_{1}+\frac{1}{2 \delta \varphi_{v}} \frac{m_{u}}{m}\right) y+\frac{m}{2 \varphi_{v}} z+\frac{1}{2 \delta \varphi_{v}} \rho, \\
& \rho_{1}=\left(b_{1}^{\prime}-\frac{1}{2 \delta \psi_{v}} \frac{m_{u}}{m}\right) y+\frac{m}{2 \psi_{v}} z-\frac{1}{2 \delta \psi_{v}} \rho, \\
& \sigma_{1}=P_{1}^{\prime} y+Q_{1}^{\prime} z+R_{1}^{\prime} \rho+S_{1}^{\prime} \sigma,
\end{aligned}
$$

* Cf. E. J. Wilczynski, Brussels Paper, p. 20, equation 17.

$\dagger$ E. J. Wilczynski, Projective differential geometry of curved surfaces, these $\mathrm{T}$ r a n s a c t i o n s, vol. 8 (1907), p. 248.

$\ddagger$ E. J. Wilczynski, Brussels Paper, p. 23, equation 23. 
where

$$
\begin{aligned}
4 \delta^{2} \varphi_{v} \psi_{v} P_{1}=-a-\frac{m_{u}}{m}\left[\delta\left(\frac{\varphi_{v v}}{\varphi_{v}}-\frac{\psi_{v v}}{\psi_{v}}\right)+\right. & \left.2 B_{1}\right] \\
& +2 \delta^{2} \varphi_{v} \psi_{v}\left[\left(a_{1}\right)_{v_{1}}+\left(b_{1}^{\prime}\right)_{u_{1}}+2 a_{1} b_{1}^{\prime}\right]
\end{aligned}
$$

$$
\begin{aligned}
4 \varphi_{v} \psi_{v} Q_{1} & =m\left(\frac{\varphi_{v v}}{\varphi_{v}}+\frac{\psi_{v v}}{\psi_{v}}-6 C_{1}+2 \frac{d_{v}}{d}+2 \frac{n_{v}}{n}\right) \\
4 \delta^{2} \varphi_{v} \psi_{v} R_{1} & =-\delta\left(\frac{\varphi_{v v}}{\varphi_{v}}-\frac{\psi_{v v}}{\psi_{v}}\right)-2 B_{1} \\
4 \varphi_{v} \psi_{v} \mathrm{~S}_{1} & =2 m
\end{aligned}
$$

Solving (15) for $y, z, \rho$ and $\sigma$ gives

$$
\begin{aligned}
& y=y_{1}, \\
& z=-\left(\frac{a_{1} \varphi_{v}+b_{1}^{\prime} \psi_{v}}{m}\right) y_{1}+\frac{\varphi_{v}}{m} z_{1}+\frac{\psi_{v}}{m} \rho_{1}, \\
& \rho=-\delta\left(a_{1} \varphi_{v}-b_{1}^{\prime} \psi_{v}+\frac{m_{u}}{\delta m}\right) y_{1}+\delta \varphi_{v} z_{1}-\delta \psi_{v} \rho_{1}, \\
& \sigma=P_{1} y+Q_{1} z+R_{1} \rho+S_{1} \sigma,
\end{aligned}
$$

where

$$
\begin{aligned}
& P_{1}=\frac{2 \varphi_{v} \psi_{v}}{m}\left[-P_{1}^{\prime}+\left(\frac{a_{1} \varphi_{v}+b_{1}^{\prime} \psi_{v}}{m}\right) Q_{1}^{\prime}+\delta\left(a_{1} \varphi_{v}-b_{1}^{\prime} \psi_{v}+\frac{m_{u}}{\delta m}\right) R_{1}^{\prime}\right] \\
& Q_{1}=\frac{2 \varphi_{v} \psi_{v}}{m}\left(-\frac{\varphi_{v}}{m} Q_{1}^{\prime}-\delta \varphi_{v} R_{1}^{\prime}\right), \\
& R_{1}=\frac{2 \varphi_{v} \psi_{v}}{m}\left(-\frac{\psi_{v}}{m} Q_{1}^{\prime}+\delta \psi_{v} R_{1}^{\prime}\right), \\
& S_{1}=\frac{2 \varphi_{v} \psi_{v}}{m} .
\end{aligned}
$$

From equations (11) and (12) we find

$$
\begin{aligned}
& a_{1} \varphi_{v}+b_{1}^{\prime} \psi_{v}=\frac{1}{2}\left(\frac{\varphi_{v v}}{\varphi_{v}}+\frac{\psi_{v v}}{\psi_{v}}-2 C_{1}+2 \frac{\delta_{v}}{\delta}\right), \\
& a_{1} \varphi_{v}-b_{1}^{\prime} \psi_{v}+\frac{m_{u}}{\delta m}=\frac{1}{2}\left(\frac{\varphi_{v v}}{\varphi_{v}}-\frac{\psi_{v v}}{\psi_{v}}-\frac{2}{\delta} B_{1}\right),
\end{aligned}
$$


so that $P_{1}, \ldots, S_{1}$ may be written in the form

$$
\begin{aligned}
& P_{1}=\frac{1}{m}\left\{\frac{\varphi_{v v}}{\varphi_{v}} \frac{\psi_{v v}}{\psi_{v}}-\varphi_{v} \psi_{v}\right. {\left[\left(a_{1}\right)_{v_{1}}+\left(b_{1}^{\prime}\right)_{u_{1}}+2 a_{1} b_{1}^{\prime}\right]+\frac{1}{2} \frac{\varphi_{v v}}{\varphi_{v}}\left[\frac{m_{u}}{\delta m}-\frac{\partial}{\partial v}\left(\left(f+\log \frac{m}{n}\right)\right]\right.} \\
&-\frac{1}{2} \frac{\psi_{v v}}{\psi_{v}}\left[\frac{m_{u}}{\delta m}+\frac{\partial}{\partial v}\left(f+\log \frac{m}{n}\right)\right]-\frac{m}{d}\left(\frac{a}{2}+\frac{m_{u}}{m} B_{1}+B_{1}^{2}\right)\left.+\frac{1}{2}\left(\frac{d_{v}}{d}+\frac{n_{v}}{n}-3 C_{1}\right)\left(\frac{d_{v}}{d}-\frac{m_{v}}{m}-2 C_{1}\right)\right\}, \\
& Q_{1}=-\frac{\varphi_{v}}{m}\left[\frac{\psi_{v v}}{\psi_{v}}-G_{1}-\frac{1}{2} \frac{\partial}{\partial v}\left(2 f+\log \frac{m d}{n^{2}}\right)\right], \\
& R_{1}=-\frac{\psi_{v}}{m}\left[\frac{\varphi_{v v}}{\varphi_{v}}-F_{1}-\frac{1}{2} \frac{\partial}{\partial v}\left(2 f+\log \frac{m d}{n^{2}}\right)\right], \\
& S_{1}=\frac{2 \varphi_{v} \psi_{v}}{m} .
\end{aligned}
$$

Let the coördinates of a point with respect to the tetrahedron $P_{y} P_{z} P_{p} P_{\sigma}$ of the congruence be $x_{1}, x_{2}, x_{3}, x_{4}$, and let the coördinates of the same point with respect to the tetrahedron $P_{y_{1}} P_{z_{1}} P_{\rho_{1}} P_{\sigma_{1}}$ of the surface $S_{y}$ be $x_{1}{ }^{\prime}, x_{2}{ }^{\prime}, x_{3}{ }^{\prime}, x_{4}{ }^{\prime}$; then

$$
\omega\left(x_{1}^{\prime} y_{1}+x_{2}^{\prime} z_{1}+x_{3}^{\prime} \rho_{1}+x_{4}^{\prime} \sigma_{1}\right)=x_{1} y+x_{2} z+x_{3} \rho+x_{4} \sigma
$$

where $\omega$ is a factor of proportionality which may be a function of $u$ and $v$.

Substitution in (21) of the value of $y, z, \rho$ and $\sigma$ as given in (17) gives an identity in $y_{1}, z_{1}, \rho_{1}$, and $\sigma_{1}$ and leads to the following equations for the transformation of coördinates:

$$
\begin{aligned}
& \omega x_{1}^{\prime}=x_{1}-\left(\frac{a_{1} \varphi_{v}+b_{1}^{\prime} \psi_{v}}{m}\right) x_{2}-\delta\left(a_{1} \varphi_{v}-b_{1}^{\prime} \psi_{v}+\frac{m_{u}}{\delta m}\right) x_{3}+P_{1} x_{4}, \\
& \omega x_{2}^{\prime}=\frac{\varphi_{v}}{m} x_{2}+\delta \varphi_{v} x_{3}+Q_{1} x_{4}, \\
& \omega x_{3}^{\prime}=\frac{\psi_{v}}{m} x_{2}-\delta \psi_{v} x_{3}+R_{1} x_{4}, \\
& \omega x_{4}^{\prime}=\frac{2 \varphi_{v} \psi_{v}}{m} x_{4} .
\end{aligned}
$$

The computation for the second focal sheet is quite similar. The differential equations which characterize $S_{*}$ are

$$
\begin{aligned}
z_{v v}-\frac{c^{\prime}}{n} z_{u u} & =b^{\prime} z+\left(\frac{a^{\prime}}{n}-\frac{c^{\prime}}{n} \frac{n_{u}}{n}\right) z_{u}+d^{\prime} z_{v}, \\
z_{u v} & =m n z+\frac{n_{v}}{n} z_{u} .
\end{aligned}
$$


Make the transformation

where

$$
u_{2}=\lambda(u, v), v_{2}=\mu(u, v),
$$

$$
\lambda_{v}=\epsilon \lambda_{u}, \quad \mu_{v}=-\epsilon \mu_{u}, \quad \epsilon^{2}=-\frac{c^{\prime}}{n},
$$

in order to make the asymptotic curves of $S_{z}, u_{2}=$ const. and $v_{2}=$ const., parametric. Then one obtains

$$
\begin{aligned}
& z_{u}=\lambda_{u} z_{u_{2}}+\mu_{u} z_{v_{2}}, z_{v}=\epsilon\left(\lambda_{u_{2}} z_{u_{2}}-\mu_{u} z_{v_{2}}\right) \\
& z_{u u}=\lambda_{u u}^{2} z_{u_{2} u_{2}}+2 \lambda_{u} \mu_{u} z_{u_{2} v_{2}}+\mu_{u}^{2} z_{v_{v} v_{2}}+\lambda_{u u} z_{u_{2}}+\mu_{u u} z_{v_{2}} \\
& z_{u v}=\epsilon\left(\lambda_{u}^{k} z_{u_{2} u_{2}}-\mu_{u}^{2} z_{v_{2} v_{2}}\right)+\left(\epsilon \lambda_{u u}+\epsilon_{u} \lambda_{u}\right) z_{u_{2}}-\left(\epsilon \mu_{u u}+\epsilon_{u} \mu_{u}\right) z_{v_{2}} \\
& z_{v v}=\epsilon^{2}\left(\lambda_{u}^{2} z_{u_{2} u_{2}}-2 \lambda_{u} \mu_{u} z_{u_{2} v_{2}}+\mu_{u}^{2} z_{v_{v} v_{2}}\right)+\left(\epsilon \lambda_{u v}+\epsilon_{v} \lambda_{u}\right) z_{u_{2}} \\
& \qquad\left(\epsilon \mu_{u v}+\epsilon_{v} \mu_{u}\right) z_{v_{2}} .
\end{aligned}
$$

Equations (24) substituted in (23) give the differential equations of $S_{z}$ referred to its asymptotic lines. These equations are

$$
\begin{aligned}
& z_{u_{2} u_{2}}+2 a_{2} z_{u_{2}}+2 b_{2} z_{v_{2}}+c_{2} z=0, \\
& z_{v_{2} v_{2}}+2 a_{2}^{\prime} z_{u_{2}}+2 b_{2}^{\prime} z_{v_{2}}+c_{2}^{\prime} z=0,
\end{aligned}
$$

where

$$
\begin{aligned}
2 a_{2} & =\frac{1}{\lambda_{u}}\left(\frac{\lambda_{u u}}{\lambda_{u}}+F_{2}-\frac{n_{v}}{\epsilon n}+\frac{\epsilon_{u}}{\epsilon}\right), 2 a_{2}^{\prime}=\frac{\lambda_{u}}{\mu_{u}^{2}} F_{2,}, \\
2 b_{2} & =\frac{\mu_{u}}{\lambda_{u}^{2}} G_{2}, 2 b_{2}^{\prime}=\frac{1}{\mu_{u}}\left(\frac{\mu_{u u}}{\mu_{u}}+G_{2}+\frac{n_{v}}{\epsilon n}+\frac{\epsilon_{u}}{\epsilon}\right), \\
c_{2} & =-\frac{1}{4 \epsilon^{2} \lambda_{u}^{2}}\left(b^{\prime}+2 \epsilon m n\right), c_{2}^{\prime}=-\frac{1}{4 \epsilon^{2} \mu_{u}^{2}}\left(b^{\prime}-2 \epsilon m n\right),
\end{aligned}
$$

where

$$
\begin{aligned}
& B_{2}=-\frac{1}{4}\left(\frac{a^{\prime}}{c^{\prime}}-\frac{n_{u}}{n}-\frac{\epsilon_{u}}{\epsilon}\right)=\frac{1}{4} \frac{\partial}{\partial u}\left(f+\log \epsilon^{3} n^{2}\right)=\frac{1}{4}\left(f_{u}+\frac{3}{2} \frac{c^{\prime}{ }_{u}}{c^{\prime}}+\frac{1}{2} \frac{n_{u}}{n}\right), \\
& C_{2}=\frac{1}{4}\left(d^{\prime}-2 \frac{n_{v}}{n}-\frac{\epsilon_{v}}{\epsilon}\right)=\frac{1}{4} \frac{\partial}{\partial v}\left(f-\log \epsilon n^{2}\right)=\frac{1}{4}\left(f_{v}-\frac{1}{2} \frac{c^{\prime}}{c^{\prime}}-\frac{3}{2} \frac{n_{v}}{n}\right), \\
& F_{2}=-\frac{1}{\epsilon} C_{2}-B_{2}, \\
& G_{2}=\frac{1}{\epsilon} C_{2}-B_{2} .
\end{aligned}
$$

The fundamental relative semicovariants of $S_{z}$ are

$$
\begin{aligned}
& y_{2}=z, \quad z_{2}=z_{u_{2}}+a_{2} z, \quad \rho_{2}=z_{v_{2}}+b_{2}^{\prime} z \\
& \sigma_{2}=z_{u_{2 v_{2}}}+b_{2}^{\prime} z_{u_{2}}+a_{2} z_{v_{2}}^{\prime}+\frac{1}{2}\left[\left(a_{2}\right)_{v_{2}}+\left(b_{2}^{\prime}\right)_{u_{2}}+2 a_{2} b_{2}^{\prime}\right] z .
\end{aligned}
$$


Using (14) and (28) one obtains

$$
\begin{aligned}
& y=-\left(\frac{a_{2} \lambda_{u}+b_{2}^{\prime} \mu_{u}}{n}\right) y_{2}+\frac{\lambda_{u}}{n} z_{2}+\frac{\mu_{u}}{n} \rho_{2}, \\
& z=y_{2}, \\
& \rho=P_{2} y_{2}+Q_{2} z_{2}+R_{2} \rho_{2}+S_{2} \sigma_{2}, \\
& q=-\epsilon\left(a_{2} \lambda_{u}-b_{2}^{\prime} \mu_{u}+\frac{n_{v}}{\epsilon n}\right) y_{2}+\epsilon \lambda_{u} z_{2}-\epsilon \mu_{u} \rho_{2},
\end{aligned}
$$

where

$$
\begin{aligned}
P_{2}=\frac{1}{n}\{ & \frac{\lambda_{u u}}{\lambda_{u}} \frac{\mu_{u u}}{\mu_{u}}-\lambda_{u} \mu_{u}\left[\left(a_{2}\right)_{v_{2}}+\left(b_{2}^{\prime}\right)_{u_{2}}+2 a_{2} b_{2}^{\prime}\right] \\
& +\frac{1}{2} \frac{\lambda_{u u}}{\lambda_{u}}\left[\frac{n_{v}}{\epsilon n}-\frac{\partial}{\partial u}\left(f+\log \frac{n}{m}\right)\right]-\frac{1}{2} \frac{\mu_{u u}}{\mu_{u}}\left[\frac{n_{v}}{\epsilon n}+\frac{\partial}{\partial v}\left(f+\log \frac{n}{m}\right)\right. \\
& \left.\quad-\frac{n}{c^{\prime}}\left(\frac{b^{\prime}}{2}+\frac{n_{v}}{n} C_{2}+C_{2}^{2}\right)+\frac{1}{2}\left(\frac{c_{u}^{\prime}}{c^{\prime}}+\frac{m_{u}}{m}-3 B_{2}\right)\left(\frac{c_{u}^{\prime}}{c^{\prime}}-\frac{n_{u}}{n}-2 B_{2}\right)\right\}, \\
Q_{2}=-\frac{\lambda_{u}}{\lambda} & {\left[\frac{\mu_{u u}}{\mu}-G_{2}-\frac{1}{2} \frac{\partial}{\partial u}\left(2 f+\log \frac{c^{\prime} n}{m^{2}}\right)\right], } \\
R_{2}=-\frac{\mu_{u}}{\mu} & {\left[\frac{\lambda_{u u}}{\lambda_{u}}-F_{2}-\frac{1}{2} \frac{\partial}{\partial u}\left(2 f+\log \frac{c^{\prime} u}{m^{2}}\right)\right], } \\
S_{2}= & \frac{2 \lambda_{u} \mu_{u}}{n} .
\end{aligned}
$$

Let the coördinates of a point with respect to the tetrahedron $P_{y} P_{z} P_{p} P_{\sigma}$ of the congruence be $x_{1}, x_{2}, x_{3}, x_{4}$, and let the coördinates of this point with respect to the tetrahedron $P_{y_{2}} P_{z_{2}} P_{\rho_{2}} P_{\sigma 2}$ of the surface $S_{x}$ be $x_{1}^{\prime \prime}, x_{2}^{\prime \prime}, x_{3}^{\prime \prime}, x_{4}^{\prime \prime}$; then

$$
\pi\left(x_{1}^{\prime \prime} y_{2}+x_{2}^{\prime \prime} z_{2}+x_{3}^{\prime \prime} \rho_{2}+x_{4}^{\prime \prime} \sigma_{2}\right)=x_{1} y+x_{2} z+x_{3} \rho+x_{4} \sigma
$$

where $\pi$ is a factor of proportionality which may be a function of $u$ and $v$. If the values of $y, z, \rho$ and $\sigma$ as given in (29) are substituted in (31), there results an identity in $y_{2}, z_{2}, \rho_{2}$, and $\sigma_{2}$ which leads to the following relations:

$$
\begin{aligned}
\pi x_{1}^{\prime \prime} & =-\left(\frac{a_{2} \lambda_{u}+b_{2}^{\prime} \mu_{u}}{n} x_{1}+x_{2}+P_{2} x_{3}-\epsilon\left(a_{2} \lambda_{u}-b_{2}^{\prime} \mu_{u}+\frac{n_{v}}{\epsilon n}\right) x_{4},\right. \\
\pi x_{2}^{\prime \prime} & =\frac{\lambda_{u}}{n} x_{2}+Q_{2} x_{3}+\epsilon \lambda_{u} x_{4}, \\
\pi x_{3}^{\prime \prime} & =\frac{\mu_{u}}{n} x_{1}+R_{2} x_{3}-\epsilon \mu_{u} x_{4}, \\
\pi x_{4}^{\prime \prime} & =\frac{2 \lambda_{u} \mu_{u}}{n} x_{3} .
\end{aligned}
$$


3. EQUATIONS OF THE EIGHT LINES REFERRED TO THE TETRAHEDRON OF THE CONGRUENCE. A STUDY OF THE POSSIBLE COINCIDENCES

The equations of the directrix of the first kind of $P_{y}$ referred to $P_{y_{1}} P_{z_{1}} P_{p_{1}}$ $P_{\sigma_{1}}$ are $^{*}$

$$
x_{4}^{\prime}=0, \quad x_{1}^{\prime}+\frac{\left(a_{1}^{\prime}\right)_{u_{2}}}{2 a_{1}^{\prime}}+\frac{\left(b_{1}\right)_{v_{1}}}{2 b_{1}} x_{3}^{\prime}=0
$$

If the values of $x_{1}, \ldots, x_{4}$ as given by (22) are substituted in (33), the equations of the directrix of the first kind of $P_{y}$ referred to $P_{y} P_{z} P_{\rho} P_{\sigma}$ are found to be

$$
\begin{aligned}
x_{4}=0, & \\
x_{1}+\frac{1}{m}\left[-\left(a_{1} \varphi_{v}+b_{1}^{\prime} \psi_{v}\right)\right. & \left.+\frac{\left(a_{1}^{\prime}\right)_{u_{1}}}{2 a_{1}^{\prime}} \varphi_{v}+\frac{\left(b_{1}\right)_{v_{1}}}{2 b_{1}} \psi_{v}\right] x_{2} \\
& -\delta\left[a_{1} \varphi_{v}-b_{1}^{\prime} \psi_{v}-\frac{m_{u}}{\delta m}-\frac{\left(a_{1}^{\prime}\right)_{u}}{2 a_{1}^{\prime}} \varphi_{v}+\frac{\left(b_{1}\right)_{v_{1}}}{2 b_{1}} \psi_{v}\right] x_{3}=0 .
\end{aligned}
$$

From equations (11) and (12) we find

$$
\begin{aligned}
& \frac{\left(a_{1}^{\prime}\right)_{u_{1}}}{2 a_{1}^{\prime}} \varphi_{v}=\frac{1}{4}\left[2 \frac{\varphi_{v v}}{\varphi_{v}}+3 \frac{\delta_{v}}{\delta}+\frac{1}{\delta} \frac{\partial}{\partial u}\left(\log F_{1}\right)+\frac{\partial}{\partial v}\left(\log F_{1}\right)\right], \\
& \frac{\left(b_{1}\right)_{v_{1}}}{2 b_{1}} \psi_{v}=\frac{1}{4}\left[2 \frac{\psi_{v v}}{\psi_{v}}+3 \frac{\delta_{v}}{\delta}-\frac{1}{\delta} \frac{\partial}{\partial u}\left(\log G_{1}\right)+\frac{\partial}{\partial v}\left(\log G_{1}\right)\right],
\end{aligned}
$$

so that the equations of the directrix of the first kind of $P_{y}\left(d_{1}^{\prime}\right)$ referred to $P_{y} P_{z} P_{p} P_{\sigma}$ become

$$
x_{4}=0, x_{1}+L_{1} x_{2}+M_{1} x_{3}=0,
$$

where

$$
\begin{aligned}
& L_{1}=\frac{1}{4 \delta m}\left[\frac{\partial}{\partial u} \log \frac{F_{1}}{G_{1}}+\delta \frac{\partial}{\partial v}\left(f+\log \delta^{5} m^{2} F_{1} G_{1}\right)\right] \\
& M_{1}=\frac{1}{4}\left[\frac{\partial}{\partial u}\left(f+\log \frac{\dot{F}_{1} G_{1}}{\delta m^{2}}\right)+\delta \frac{\partial}{\partial v} \log \frac{F_{1}}{G_{1}}\right] .
\end{aligned}
$$

The equations of the directrix of the second kind of $P_{y}$ referred to $P_{y_{1}} P_{z_{1}} P_{\rho_{1}} P_{\sigma_{1}}$ are

$$
x_{2}^{\prime}+\frac{\left(b_{1}\right)_{v_{1}}}{2 b_{1}} x_{4}^{\prime}=0, \quad x_{3}^{\prime}+\frac{\left(a_{1}^{\prime}\right)_{u_{1}}}{2 a_{1}^{\prime}} x_{4}^{\prime}=0
$$

and these equations by the use of (11), (22) and (35) give as the equations of the directrix of the second kind of $P_{y}\left(d_{1}^{\prime \prime}\right)$ referred to $P_{y} P_{z} P_{\rho} P_{\sigma}$

$$
x_{2}+S_{1} x_{4}=0, \quad x_{3}+T_{1} x_{4}=0
$$

* E. J. Wilczynski, Projective differential geometry of curved surfaces, these T $\mathrm{r}$ a $\mathrm{n}$ s a ct i o n s, vol. 9 (1908), p. 95, equations $(70 a)$ and (70b). 
where

$$
\begin{aligned}
& S_{1}=\frac{1}{4 \delta}\left[\frac{\partial}{\partial u} \log \frac{F_{1}}{G_{1}}+\delta \frac{\partial}{\partial v}\left(3 f+\log \frac{\delta^{7} m^{2}}{n^{4}} F_{1} G_{1}\right)\right] \\
& T_{1}=\frac{1}{4 \delta^{2} m}\left[\frac{\partial}{\partial u}\left(f-\log \delta m^{2} F_{1} G_{1}\right)-\delta \frac{\partial}{\partial v} \log \frac{F_{1}}{G_{1}}\right] .
\end{aligned}
$$

In a similar way one may obtain the equations of the directrix of the first kind of $P_{\mathbf{z}}\left(d_{2}^{\prime}\right)$ referred to $P_{y} P_{\mathbf{z}} P_{p} P_{\sigma} ;$ they are

$$
x_{3}=0, \quad L_{2} x_{1}+x_{2}+M_{2} x_{4}=0,
$$

where

$$
\begin{aligned}
& L_{2}=\frac{1}{4 n}\left[\frac{\partial}{\partial u}\left(f+\log \epsilon^{5} n^{2} F_{2} G_{2}\right)+\frac{1}{\epsilon} \frac{\partial}{\partial v} \log \frac{F_{2}}{G_{2}}\right], \\
& M_{2}=\frac{\epsilon}{4}\left[\frac{\partial}{\partial u} \log \frac{F_{2}}{G_{2}}+\frac{1}{\epsilon} \frac{\partial}{\partial v}\left(f+\log \frac{F_{2} G_{2}}{\epsilon n^{2}}\right)\right] ;
\end{aligned}
$$

and the equations of the directrix of the second kind of $P_{z}\left(d_{2}^{\prime \prime}\right)$ referred to $P_{y} P_{z} P_{p} P_{\sigma}$, namely

$$
x_{1}+S_{2} x_{3}=0, \quad T_{2} x_{3}+x_{4}=0,
$$

where

$$
\begin{aligned}
& S_{2}=\frac{1}{4}\left[\frac{\partial}{\partial u}\left(3 f+\log \frac{\epsilon^{7} n^{2}}{m^{4}} F_{2} G_{2}\right)+\frac{1}{\epsilon} \frac{\partial}{\partial v} \log \frac{F_{2}}{G_{2}}\right], \\
& T_{2}=\frac{1}{4 \epsilon n}\left[-\frac{\partial}{\partial u} \log \frac{F_{2}}{G_{2}}+\frac{1}{\epsilon} \frac{\partial}{\partial v}\left(f-\log \epsilon n^{2} F_{2} G_{2}\right] .\right.
\end{aligned}
$$

The equations of the axis of $P_{y}\left(x_{1}\right)$ are*

$$
x_{3}=0, \quad x_{2}+K_{1} x_{4}=0,
$$

where

$$
K_{1}=f_{v}+\frac{d_{v}}{d}-\frac{n_{v}}{n} .
$$

The equations of the ray of $P_{y}\left(r_{1}\right)$ are $\dagger$

$$
x_{1}=0, \quad x_{4}=0 \text {. }
$$

The equations of the axis of $P_{2}\left(x_{2}\right)$ are

$$
x_{4}=0, \quad x_{1}+K_{2} x_{3}=0 \text {, }
$$

* E. J. Wilczynski, The general theory of congruences, these T r a n s a c t i o n s, vol. 16 (1915), p. 313.

$\dagger$ Loc. cit., p. 318 
where

$$
K_{2}=f_{u}+\frac{c^{\prime}{ }_{u}}{c^{\prime}}-\frac{m_{u}}{m}
$$

and of the ray $\left(r_{2}\right)$

$$
x_{2}=0, \quad x_{3}=0 .
$$

Certain relations between the relative invariants $B_{1}, C_{1}, B_{2}, C_{2}, K_{1}, \ldots$, $T_{2}$ will be useful in the sequel, and are given here. They may be verified by computation:

$$
\begin{aligned}
& S_{1}-m L_{1}=K_{1}-2 C_{1}, \\
& S_{2}-n L_{2}=K_{2}-2 B_{2}, \\
& 2 B_{1}-M_{1}=\delta^{2} m T_{1}, \\
& 2 C_{2}-M_{2}=\epsilon^{2} n T_{2} .
\end{aligned}
$$

The points $P_{y}$ and $P_{z}$ will not coincide for all values of $u$ and $v$, since we are excluding the case of congruences with coincident focal sheets. $*$ Therefore the tangent plane to $S_{y}$ at $P_{y}\left(x_{4}=0\right)$ is in general distinct from the tangent plane to $S_{z}$ at $P_{z}\left(x_{3}=0\right)$. From these two facts one finds that certain pairs of lines cannot coincide (this may also be seen from the equations of the various lines); in fact, there remain only twelve possible coincidences. Let $\left(d_{1}^{\prime} d_{2}\right)$ denote the coincidence of the two lines $\dot{d}_{1}^{\prime}$ and $d_{2}^{\prime \prime}$, etc.; then the twelve possible coincidences and the corresponding analytic conditions are as follows:

$$
\begin{array}{ll}
\left(d_{1}^{\prime} d_{2}^{\prime \prime}\right): L_{1}=T_{2}=S_{2}-M_{1}=0 ; & \left(d_{2}^{\prime} d_{1}^{\prime \prime}\right): L_{2}=T_{1}=S_{1}-M_{2}=0 \\
\left(r_{1} x_{2}\right): K_{2}=0 ; & \left(r_{2} x_{1}\right): K_{1}=0 ; \\
\left(d_{1}^{\prime} r_{1}\right): L_{1}=M_{1}=0 ; & \left(d_{2}^{\prime} r_{2}\right): L_{2}=M_{2}=0 ; \\
\left(d_{1}^{\prime} x_{2}\right): L_{1}=M_{1}-K_{2}=0 ; & \left(d_{2}^{\prime} x_{1}\right): L_{2}=M_{2}-K_{1}=0 \\
\left(d_{1}^{\prime \prime} x_{1}\right): T_{1}=S_{1}-K_{1}=0 ; & \left(d_{2}^{\prime \prime} x_{2}\right): T_{2}=S_{2}-K_{2}=0 ; \\
\left(d_{1}^{\prime \prime} r_{2}\right): S_{1}=T_{1}=0 ; & \left(d_{2}^{\prime \prime} r_{1}\right): S_{2}=T_{2}=0 ;
\end{array}
$$

where the values of $K_{1}$, etc., are given in the preceding section.

The axis and ray of a point on either focal sheet of a congruence correspond by duality and the two directrices of such a point also correspond by duality. So the coincidences $\left(d_{1}^{\prime \prime} x_{1}\right),\left(d_{2}^{\prime \prime} x_{2}\right),\left(d_{1}^{\prime \prime} r_{2}\right)$ and $\left(d_{2}^{\prime \prime} r_{1}\right)$ will be called the duals of $\left(d_{1}^{\prime} r_{1}\right),\left(d_{2}^{\prime} r_{2}\right),\left(d_{1}^{\prime} x_{2}\right)$ and $\left(d_{2}^{\prime} x_{1}\right)$, respectively. If a congruence has a coincidence then the congruence obtained from the given one by duality has the dual coincidence. In fact, the substitution which transforms the coefficients of a congruence into the coefficients of the dual congruence transforms the conditions for any coincidence into the conditions for the dual coincidence. This fact enables one to reduce the amount of calculation involved in the study of the possible combina-

* E. J. Wilczynski, Brussels Paper, pp. 11-12. 
tions of the twelve coincidences. For example, the canonical form and properties of the congruences characterized by the coincidences $\left(d_{1}^{\prime} r_{1}\right)$ and $\left(d_{2}^{\prime} r_{2}\right)$ gives by a substitution alone the canonical form and properties of the congruences characterized by the coincidences $\left(d_{1}^{\prime \prime} x_{1}\right)$ and $\left(d_{2}^{\prime \prime} x_{2}\right)$.

It is not evident that there exists a congruence which has any one of the twelve (apparently) possible coincidences, for there might be no solution to the integrability conditions and the corresponding coincidence conditions taken together. However, the congruence which has the coefficients

$$
m=n=c^{\prime}=d=1, \quad a^{\prime}=b=c=d^{\prime}=0, \quad a=-b^{\prime}=\text { const., }
$$

satisfies the conditions for all the coincidences, so that there surely exists at least this one solution for any group of equations obtained by using any combination of the twelve coincidences. The congruences characterized by (52) will be referred to as coincidence congruences in the remainder of this article. Since $m \neq 0, n \neq 0$, neither of the focal sheets of a coincidence congruence degenerates into a curve, and since $c^{\prime} \neq 0, d \neq 0$ neither of its focal sheets is developable. * We shall throughout çonsider only such $\left(m n c^{\prime} d \neq 0\right)$ non-degenerate congruences. The coincidence congruences have four $\left(d_{1}^{\prime}, d_{1}^{\prime \prime}, d_{2}^{\prime}, d_{2}^{\prime \prime}\right)$ of their eight lines indeterminate, so that the coincidences in this case have but little meaning. We shall show that certain sets of coincidences give rise to congruences for which the eight lines are determinate, while for certain other sets some of the eight lines are of necessity indeterminate.

4. THE PROPERTIES AND CANONICAL FORM OF CONGRUENCES POSSESSING COINCIDENCES $\left(d_{1}^{\prime} r_{1}\right)$ AND $\left(d_{1}^{\prime \prime} r_{2}\right)$

The conditions which give these coincidences are $L_{1}=M_{1}=S_{1}=T_{1}=0$. From these equations and (51), we find

$$
B_{1}=0, \quad K_{1}=2 C_{1}, \quad F_{1}=G_{1}=-C_{1} .
$$

Using (53) in $L_{1}=0$ and $M_{1}=0$ gives

$$
\frac{\partial}{\partial v}\left(2 f+\log \frac{d^{5}}{m} C_{1}^{4}\right)=0, \frac{\partial}{\partial u} \log C_{1}=0,
$$

whence by integration

$$
C_{1}=V(v), \frac{d^{5}}{m} C_{1}^{4} e^{2 f}=U(u)
$$

* E. J. Wilczynski, Brussels Paper, p. 28. 
where $V(v)$ is an arbitrary function of $v$ alone, and $U(u)$ is an arbitrary function of $u$ alone.*

Integration of $C_{1}=V(v)$ and $B_{1}=0$, using (11), gives

$$
m d^{3} e^{2 f}=V_{1}(v) U_{1}(u), \quad m d^{3} e^{-2 f} V_{2}(v)
$$

where $V_{1}(v)$ depends upon $V(v)$. From (54) and (55), one finds that

$$
e^{4 f}=\frac{U_{1}^{2} V_{1}^{6}}{U V_{2}}
$$

so that $f_{u v}=0$. Consider the most general transformation (3) which leaves the set (1) invariant; since $\overline{\left(e^{f}\right)}=\frac{K e^{f}}{\lambda^{2} \mu^{2} \alpha_{u} \beta_{v}}$, we can choose $\lambda, \mu, \alpha_{u}, \beta_{v}$ and $K$ in such a way as to make

$$
e^{f}=1, \quad f=0,
$$

and this relation will be left unaltered by the subgroup of (3) for which $\lambda=$ $\frac{c_{1}}{\sqrt{\alpha_{u}}}, \mu=\frac{c_{2}}{\sqrt{\beta_{v}}}$, where $c_{1}$ and $c_{2}$ are arbitrary constants. $\dagger$

From (55) and (56) we find that $\frac{d}{m}=\sqrt{\frac{U_{1} V_{1}}{V_{2}}}$; since $\left(\frac{d}{m}\right)=\frac{\beta_{v}^{2}}{\alpha_{u}^{2}} \frac{d}{m}$, we can make $d=m$, and thereafter use the subgrnup of (3) for which $\alpha_{u}=\beta_{v}=c_{1}$, $\lambda=c_{2}, \mu=c_{3}$ where $c_{1}, c_{2}$ and $c_{3}$ are arbitrary constants.

We now have, using the fifth integrability condition,

$$
f=0, \quad d=m, \quad c^{\prime}=n .
$$

These values substituted in (54) give $m C_{1}^{\prime}=\varphi(u)$; moreover we find by calculation that $C_{1}=\frac{1}{2} \frac{m_{v}}{m}$, and from $B_{1}=0$ that $m=\psi(v)$ so that $\varphi(u)=\frac{1}{2} m_{v}$ while $m=\psi(v)$. Then $m=l_{2} v+l_{1}$ and $C_{1}=\frac{l_{2}}{2\left(l_{2} v+l_{1}\right)}$.

\footnotetext{
* In the remainder of this article, arbitrary functions of $u$ alone or of $v$ alone are indicated by this same notation.

† Cf. E. J. Wilczynski, Brussels Paper, pp. 42-3. Similar transformations will often be made in the remainder of this article; in every case, the sub-group of (3) which is available for further transformations will be indicated.
} 
There are two cases to consider.

First, $l_{2}=0$; then $m=$ const. and we find that the transformations at our disposal enable us to make

$$
d=m=c^{\prime}=n=1 \text {. }
$$

Using (58) the integrability conditions give

$$
a^{\prime}=b=0, \quad c=d^{\prime}=0, \quad b=-a=\text { const. }
$$

and (57), (58) and (59) characterize the coincidence congruences.

Second, $l_{2} \neq 0$; now $\overline{(m)}=\frac{c_{3}}{c_{1} c_{2}} m_{1}$ so we can make $l_{2}=1$, and thereafter use the subgroup of (3) for which

$$
\alpha_{u}=\beta_{v}=c_{1}, \quad \lambda=c_{2}, \quad \mu=c_{1} c_{2}
$$

Now $d=m=v+l_{1}, 2 C_{1}=\frac{1}{v+l_{1}}=K_{1}=\frac{\partial}{\partial v} \log \frac{d}{n}$ whence $c^{\prime}=n=U_{3}(u)$. The sixth and seventh integrability conditions now become $a+b^{\prime}=0$ and $n_{u u}$ $+n_{v v}=0$ so that $n=c^{\prime}=l_{3} u+l_{2}$; but since $\overline{\left(c^{\prime}\right)}=\frac{1}{c_{1}^{2}} c^{\prime}$ we can make $l_{3}=$ 1.* Using

$$
f=c=d^{\prime}=0, \quad d=m=v+l_{1}, \quad c^{\prime}=n=u+l_{2}
$$

in the integrability conditions, we obtain

$$
a^{\prime}=b=-1, \quad b^{\prime}=-a,
$$

where $a_{v}=2\left(v+l_{1}\right), a_{u}=-2\left(u+l_{2}\right)$, so that

$$
a=v^{2}-u^{2}+2\left(l_{1} v-l_{2} u\right)+l_{3},
$$

where $l_{1}, l_{2}$ and $l_{3}$ are arbitrary constants.

If we use the values given by (60), (61) and (62), we obtain $L_{2}=M_{2}=S_{2}$ $=T_{2}=0, K_{1}=\frac{1}{v+l_{1}}, K_{2}=\frac{1}{u+l_{2}}$, so that these congruences possess the coin-

* If $l_{3}=0$, this cannot be done; but as in the first case above, we can show that if $l_{3}=0$ the congruence is a coincidence congruence. 
cidences $\left(d_{1}^{\prime} d_{2}^{\prime \prime}\right),\left(d_{2}^{\prime} d_{1}^{\prime \prime}\right),\left(d_{1}^{\prime \prime} r_{2}\right)$ and $\left(d_{2}^{\prime \prime} r_{1}\right)$ in addition to the given pair. To recapitulate: If a non-degenerate congruence possesses the coincidences $\left(d_{1}^{\prime} r_{1}\right)$ and $\left(d_{1}^{\prime \prime} r_{2}\right)$ it is either a coincidence congruence which possesses all twelve coincidences, or else it is a congruence whose canonical form is

$$
\begin{aligned}
f=c=d^{\prime}=0, \quad d=m=v+l_{1}, & c^{\prime}=n=u+l_{2}, \\
a^{\prime}=b=-1, & a=-b^{\prime}=v^{2}-u^{2}+2\left(l_{1} v-l_{2} u\right)+l_{3}
\end{aligned}
$$

where $l_{1}, l_{2}$ and $l_{3}$ are arbitrary constants, and possesses also the coincidences $\left(d_{1}^{\prime} d_{2}^{\prime \prime}\right)$, $\left(d_{2}^{\prime} d_{1}^{\prime \prime}\right),\left(d_{1}^{\prime \prime} r_{2}\right)$ and $\left(d_{2}^{\prime \prime} r_{1}\right)$.

The congruences dual to (63) have the canonical form*

$$
\begin{aligned}
& f=c=d^{\prime}=0, \quad d=m=u+l_{2}, \quad c^{\prime}=n=v+l_{1}, \\
& b=a^{\prime}=0, \quad a=-b^{\prime}=v^{2}-u^{2}+2\left(l_{1} v-l_{2} u\right)+l_{3} \text {, }
\end{aligned}
$$

and possess the coincidences $\left(d_{1}^{\prime} d_{2}^{\prime \prime}\right),\left(d_{2}^{\prime} d_{1}^{\prime \prime}\right),\left(d_{2}^{\prime} x_{1}\right),\left(d_{1}^{\prime} x_{2}\right),\left(d_{1}^{\prime \prime} x_{1}\right)$ and $\left(d_{2}^{\prime \prime} x_{1}\right)$.

Since there exist congruences, other than the coincidence congruences, which possess either one of the sets of coincidences noted above, there exist congruences which possess any one of the coincidences separately. We will consider in the next section the congruences which possess the coincidences $\left(r_{1} x_{2}\right)$ and $\left(r_{2} x_{1}\right)$ and we shall show that there exist congruences, other than the coincidence congruences, which possess that pair of coincidences. Using that fact, we state that there exist congruences, besides the coincidence congruences, which possess any one of the twelve possible coincidences.

The coefficients given in (63) make $K_{1}=\frac{1}{v+l_{1}} \neq 0$; if we seek those congruences which possess the coincidence $\left(r_{2} x_{1}\right)$, which makes $K_{1}=0$, in addition to the coincidences $\left(d_{1}^{\prime} r_{1}\right)$ and $\left(d_{1}^{\prime \prime} r_{2}\right)$, we find that they are coincidence congruences. More generally the coincidence congruences are the only congruences which possess all of the twelve coincidences. This justifies the term "coincidence congruences" which we have chosen for them.

5. ThE PROPERTIES AND CANONICAL FORM OF A CONGRUENCE POSSESSING COINCIDENCES $\left(r_{1} x_{2}\right)$ AND $\left(x_{1} r_{2}\right)$

Let us consider now a congruence which has the coincidences $\left(r_{1} x_{2}\right)$ and $\left(x_{1} r_{2}\right)$. By integration of the associated conditions

$$
f_{u}+\frac{c^{\prime}{ }_{u}}{c^{\prime}}-\frac{m_{u}}{m}=0 \quad f_{v}+\frac{d_{v}}{d}-\frac{n_{v}}{n}=0
$$

* E. J. Wilczynski, Brussels Paper, pp. 24-28. 
one obtains

$$
\frac{c^{\prime}}{m}=V(v) e^{-f}, \frac{d}{n}=U(u) e^{-f}
$$

Transformations (3) enable us by a proper choice of $\lambda, \mu, \alpha_{u}, \beta_{v}$ to make $V(v)=1, U(u)=1$. We may use thereafter the sub-group of (3) given by

$$
\lambda=\frac{1}{\sqrt{\alpha_{u}}}, \mu=\frac{1}{\sqrt{\beta_{r}}}
$$

We now have

$$
c^{\prime}=m e^{-f}, \quad d=n e^{-f} .
$$

Here, as previously, we exclude congruences for which $m n c^{\prime} d=0$.

From (65) we find

$$
\begin{array}{ll}
c_{u}^{\prime}=\left(m_{u}-m f_{u}\right) e^{-f}, & c_{u u}^{\prime}=\left(m_{u u}-2 m_{u} f_{u}-m f_{u u}+m f_{u}^{2}\right) e^{-f} \\
d_{v},=\left(n_{v}-n f_{v}\right) e^{-f}, & d_{v v}=\left(n_{v v}-2 n_{v} f_{v}-n f_{v v}+n f_{v}^{2}\right) e^{-f}
\end{array}
$$

If these values are substituted in the sixth and seventh integrability conditions, we obtain the equations

$$
\begin{aligned}
& m_{u u}-m_{u} f_{u}-m a=-e^{-f}\left(n_{v v}-n_{v} f_{v}-n b^{\prime}\right), \\
& m_{u u}-m_{u} f_{u}-m a=-e^{f}\left(n_{v v}-n_{v} f_{v}-n b^{\prime}\right)
\end{aligned}
$$

whence follows either $f=0$, which means that the congruence is a $W$-congruence, * or else

$$
\begin{aligned}
& m_{u u}-m_{u} f_{u}-m a=0, \\
& n_{v v}-n_{u} f_{v}-n b^{\prime}=0 ;
\end{aligned}
$$

we shall call such congruences harmonic. $\dagger$

If $f=0$, equations (65) reduce to

$$
c^{\prime}=m, \quad d=n
$$

* E. J. Wilczynski, Brussels Paper; p. 46.

† The nets of curves $u=$ const. and $v=$ const. on $S_{y}$ and $S_{z}$ are in this case harmonic conjugate nets. See E. J. Wilczynski, Geometrical significance of isothermal conjugacy of a net of curves, A m e rica $\mathrm{n}$ ournal of $\mathrm{M}$ a th e matics, vol. 42 (1920), p. 215. 
From (67) and the integrability conditions it follows that

$$
\begin{aligned}
& f=c=d^{\prime}=0, \quad b=-n_{v}, \quad a^{\prime}=-m_{u}, \\
& m_{u u}+n_{v v}=m a+n b^{\prime}, \\
& a_{v}=3 m_{u} n+m n_{u}, \quad b_{u}^{\prime}=m_{v}+3 m n_{v} .
\end{aligned}
$$

The canonical form given here is the same as the canonical form for identically self dual congruences, or for congruences which belong to linear complexes.*

Using (66), we find

$$
\begin{aligned}
& c=f_{u}, \quad d^{\prime}=f_{v}, \quad b=-n_{v} e^{-f}, a^{\prime}=-m_{u} e^{-f}, m n\left(1-e^{-2 f}\right)-f_{u v}, \\
& m n\left(3 \frac{m_{u}}{m}+\frac{n_{u}}{n}-f_{u}\right)=\frac{m_{u u v}}{m}-\frac{m_{u u}}{m} \frac{m_{v}}{m}-\frac{m_{u v}}{m} f_{u}+\frac{m_{u}}{m} \frac{m_{v}}{m} f_{u}, \\
& m n\left(\frac{m_{v}}{m}+3 \frac{n_{v}}{n}-f_{v}\right)=\frac{n_{u v v}}{n}-\frac{n_{v v}}{n} \frac{n_{u}}{n}-\frac{n_{u v}}{n} f_{v}+\frac{n_{v}}{n} \frac{n_{u}}{n} f_{v} .
\end{aligned}
$$

The results of this section may be stated as follows:

If a non-degenerate congruence possesses the coincidences $\left(r_{1} x_{2}\right)$ and $\left(x_{1} r_{2}\right)$, it is either a $W$-congruence whose canonical form is given by (67) and (68) in which case it is identically self-dual and belongs to a linear complex; or else it is an harmonic congruence whose canonical form is given by (65), (66) and (69).

6. THE PROPERTIES AND CANONICAL FORM OF CONGRUENCES WHICH POSSESS COINCIDENCES $\left(d_{1}^{\prime} d_{2}^{\prime \prime}\right)$ AND $\left(d_{1}^{\prime \prime} d_{2}^{\prime}\right)$

We consider finally the congruences which have the two coincidences $\left(d_{1}^{\prime} d_{2}^{\prime \prime}\right)$ and $\left(d_{1}^{\prime \prime} d_{2}^{\prime}\right)$. The conditions to be satisfied are

$$
L_{1}=T_{2}=S_{2}-M_{1}=0, \quad L_{2}=T_{1}=S_{1}-M_{2}=0 .
$$

Using (51), equations (70) may be replaced by

$$
L_{1}=0, \quad \frac{\partial}{\partial u} \log \frac{F_{1}}{G_{1}}+\delta \frac{\partial}{\partial v}\left(f+\log \delta^{5} m^{2} F_{1} G_{1}\right)=0
$$

(72) $L_{2}=0, \quad \epsilon \frac{\partial}{\partial u}\left(f+\log \epsilon^{5} n^{2} F_{2} G_{2}\right)+\frac{\partial}{\partial v} \log \frac{F_{2}}{G_{2}}=0$;

(73) $M_{1}=2 B_{1}, \frac{\partial}{\partial u}\left(f+\log \frac{F_{1} G_{1}}{\delta m^{2}}\right)+\delta \frac{\partial}{\partial v} \log \frac{F_{1}}{G_{1}}=\frac{\partial}{\partial u}\left(2 f-\log \delta^{2} m^{4}\right)$;

* E. J. Wilczynski, Brussels Paper, p. 28 and p. 43. 
(74) $\quad M_{2}=2 C_{2}, \quad \epsilon \frac{\partial}{\partial u} \log \frac{F_{2}}{G_{2}}+\frac{\partial}{\partial v}\left(f+\log \frac{F_{2} G_{2}}{\epsilon n^{2}}\right)=\frac{\partial}{\partial v}\left(2 f-\log \epsilon^{2} n^{4}\right)$;

(75) $S_{1}=2 C_{2}, \frac{1}{\delta} \frac{\partial}{\partial u} \log \frac{F_{1}}{G_{1}}+\frac{\partial}{\partial v}\left(3 f+\log \frac{\delta^{7} m^{2}}{n^{4}} F_{1} G_{1}\right)=\frac{\partial}{\partial v}\left(2 f-\log \epsilon^{2} n^{4}\right)$;

(76) $S_{2}=2 B_{1}, \frac{\partial}{\partial u}\left(3 f+\log \frac{\epsilon^{7} n^{2}}{m^{4}} F_{2} G_{2}\right)+\frac{1}{\epsilon} \frac{\partial}{\partial v} \log \frac{F_{2}}{G_{2}}=\frac{\partial}{\partial u}\left(2 f-\log \delta^{2} m^{4}\right)$.

If we multiply (75) by $\delta$ and subtract (71) from the result, we find

$$
\frac{\partial}{\partial v} \log \delta^{2} \epsilon^{2} \doteq 0
$$

In a similar fashion from (72) and (76) one obtains

$$
\frac{\partial}{\partial u} \log \delta^{2} \epsilon^{2}=0
$$

so that

$$
\delta \epsilon=k, \text { where } k \text { is an arbitrary constant. }
$$

Using this relation, the six conditions reduce to the four given by (71), (72) and the two following:

$$
\begin{aligned}
& \frac{\partial}{\partial u}\left(f-\log \delta m^{2} F_{1} G_{1}\right)-\delta \frac{\partial}{\partial v} \log \frac{F_{1}}{G_{1}}=0, \\
& \frac{\partial}{\partial u} \log \frac{F_{2}}{G_{2}}-\frac{1}{\epsilon} \frac{\partial}{\partial v}\left(f-\log \epsilon n^{2} F_{2} G_{2}\right)=0 .
\end{aligned}
$$

Adding (71) and (78) gives, using (11),

$$
G_{1 u}+2 \delta F_{1} G_{1}-\delta_{v} G_{1}-\delta G_{1 v}=0
$$

while subtracting (78) from (71) gives

$$
F_{1 u}-2 \delta F_{1} G_{1}+\delta_{v} F_{1}+\delta F_{1 v}=0
$$

Adding (80) and (81) gives

$$
\frac{\partial}{\partial u}\left(F_{1}+G_{1}\right)+\left(F_{1}-G_{1}\right) \delta_{v}+\delta \frac{\partial}{\partial v}\left(F_{1}-G_{1}\right)=0,
$$


which, since $F_{1}+G_{1}=-2 C_{1}, F_{1}-G_{1}=-\frac{2}{\delta} B_{1}$, becomes

$$
C_{1 u}+B_{1 v}=0
$$

But from (11),

$$
C_{1 u}+B_{1 v}=\frac{\partial^{2}}{\partial u \partial v}(f+\log \delta)
$$

so that

$$
\frac{\partial^{2}}{\partial u \partial v}(f+\log \delta)=0
$$

By a similar calculation, from (72) and (79), one obtains

$$
\frac{\partial^{2}}{\partial u \partial v}(f+\log \epsilon)=0 \text {. }
$$

From (82), (83) and (77) it follows that $f_{u v}=0$, whence

$$
k^{2}=\delta^{2} \epsilon^{2}=\frac{c^{\prime} d}{m n}=1, \quad \text { and } k= \pm 1
$$

But $\epsilon= \pm 1 / \delta$ enters in such a way into equations (71), (72), (78) and (79) that the same set of equations results from using $k=-1$ as from using $k=1$. So there is no loss of generality in assuming $k=1$. Use the transformation group (3) to make

$$
f=\text { const., } c=d^{\prime}=0 \text {; }
$$

these relations will be left unaltered by the sub-group of (3) given by

$$
\lambda=\frac{c_{1}}{\sqrt{\alpha_{u}}}, \quad \mu=\frac{c_{2}}{\sqrt{\beta_{v}}},
$$

where $c_{1}$ and $c_{2}$ are arbitrary constants.

From (84) and (82), it follows that

$$
\delta^{2}=-\frac{d}{m}=-\varphi(u) \psi(v)
$$

then by using transformations (3) we can choose $\alpha$ and $\beta$ so as to make

$$
d=m \text {. }
$$


and thereafter use the sub-group of (3) given by

$$
\lambda=\frac{c_{1}}{c_{3}}, \quad \mu=\frac{c_{2}}{c_{3}}, \quad \alpha_{u}=\beta_{v}=C_{3}^{2}
$$

where $c_{1}, c_{2}$ and $c_{3}$ are arbitrary constants.

Since $m n-c^{\prime} d=f_{u v}=0$, we find further

$$
c^{\prime}=n
$$

These equations show that the developables of the congruence intercept isothermally conjugate nets on both sheets of the focal surface. ${ }^{*}$

Subtracting (80) from (81), and using (11) and (85), one finds

$$
m_{u u}+m_{v v}=0
$$

By a similar computation based on the two equations corresponding to (80) and (81) one obtains

$$
n_{u u}+n_{v v}=0
$$

The integrability conditions become

$$
\begin{gathered}
b=-m_{v}, \quad a^{\prime}=-n_{u}, \quad b^{\prime}=-a, \\
a_{v}=2 \frac{\partial}{\partial u} m n, \quad a_{u}=-2 \frac{\partial}{\partial v} m n .
\end{gathered}
$$

If we differentiate the second equation of (90) with respect to $v$, and the first equation with respect to $u$, and subtract one resulting equation from the other, we obtain

$$
(m n)_{u u}+(m n)_{v v}=0
$$

If we perform the differentiations indicated in (91), and use (87) and (88), we obtain

$$
\frac{m_{u}}{n_{v}}=-\frac{m_{v}}{n_{u}}=\rho
$$

* E. J. Wilczynski, General theory of congruences, these T r a n s a c t i o n s, vol. 16 (1915), p. 322 . 
where $\rho$ is a function of $u$ and $v$ which we may assume to be real, if the focal sheets of the congruence are real. From (92) we find

$$
m_{u u}=\rho_{u} n_{v}+\rho n_{u v}, \quad m_{v v}=-\rho_{v} n_{u}-\rho n_{u v},
$$

whence, by (87),

$$
\rho_{u} n_{v}-\rho_{v} n_{u}=0
$$

Again we find

$$
m_{u v}=\rho_{v} n_{v}+\rho n_{v v}=-\rho_{u} n_{u}-\rho n_{u u}
$$

whence, by (88),

$$
\rho_{u} n_{u}+\rho_{v} n_{v}=0
$$

Equations (93) and (94) are two homogeneous equations which $n_{u}$ and $n_{v}$ must satisfy. The determinant of the coefficients must be zero;*i.e., $\rho_{u}^{2}+$ $\rho_{v}^{2}=0$, whence $\rho_{u}=\rho_{v}=0$ since $\rho$ is real, and $\rho=k$, where $k$ is an arbitrary real constant. Then $k=-m_{v} / n_{u}=-b / a^{\prime}$, using (2); so by using (3) we can make $k=1$ ( $k=0$ gives the coincidence congruences) and thereafter use the subgroup of (3) for which

$$
\lambda=\mu=c_{1}, \quad \alpha_{u}=\beta_{v}=c_{2}
$$

Equation (92) becomes

$$
m_{u}=-n_{v}, \quad m_{v}=n_{u}
$$

using these values, equations (90) can be integrated and give

$$
a=n^{2}-m^{2}+k
$$

where $k$ is an arbitrary constant.

The congruences which possess the coincidences $\left(d_{1}^{\prime} d_{2}^{\prime \prime}\right)$ and $\left(d_{1}^{\prime \prime} d_{2}^{\prime}\right)$ are identical with the congruences which arise from the Riemann sphere representation of functions of a complex vairable according to one of the methods discussed

\footnotetext{
* Or else $n_{u}=n_{v}=0$; then $n=$ const. and if $n \neq 0$ we can make $n=1$ and are led to the coincidence congruences, which form a special case of the general set of congruences possessing the given coincidences.
} 
by Wilczynski.* We shall call them bidirectrix congruences since the four directrices associated in general with a line of the congruence in this case reduce to two. Thus the property of possessing coincidences $\left(d_{1}^{\prime} d_{2}^{\prime \prime}\right)$ and $\left(d_{1}^{\prime \prime} d_{2}^{\prime}\right)$ characterizes completely this set of bidirectrix congruences, discovered by Wilczynski.

Using Wilczynski's results in connection with the theorem just stated we see that a non-degenerate congruence in the real and distinct focal sheets, which possesses the coincidences $\left(d_{1}^{\prime} d_{2}^{\prime \prime}\right)$ and $\left(d_{1}^{\prime \prime} d_{2}^{\prime}\right)$, has also the following properties:

(a) it is a $W$-congruence;

(b) its developables intercept isothermally conjugate nets on both sheets of the focal surface;

(c) the asymptotic curves on both focal sheets belong to linear complexes;

(d) corresponding asymptotic curves belong to the same complex; $\dagger$

(e) the directrix curves of the two sheets of the focal surface correspond to each other;

(f) on each sheet of the focal surface, each of its axis curve tangents is conjugate to one of the ray curve tangents.

Moreover it has the canonical form

$$
c=d^{\prime}=0, d=m, c^{\prime}=n, a^{\prime}=-b=-n_{u}, a=-b^{\prime}=n^{2}-m^{2}+k,
$$

where

$$
m_{u}=n_{v}, \quad m_{v}=-n_{u}
$$

and $k$ is an arbitrary constant.

* Line geometric representations for functions of a complex variable, these $\mathrm{T} \mathrm{r}$ a $\mathrm{n} \mathrm{s}$ a ct i o n s, vol. 20 (1919), pp. 283-298.

A set of properties characteristic of a class of congruences connected with the theory of functions, these T r a n s a c t i o n s, vol. 21 (1920), pp. 409-445.

$\dagger$ This property is added by the author and may be proved as follows. Denote by $\Gamma$ the linear complex to which the asymptotic curve $v_{1}=$ const. on $S_{y}$ belongs so that the linear complex osculating $v_{1}=$ const. at any point is $\Gamma$. In view of the coincidence property, the two linear complexes osculating $u_{1}=$ const. and $v_{1}=$ const. which pass through $P_{y}$ have in common a linear congruence whose directrices are the directrices of the first kind of $P_{y}$ and $P_{z}$. All the lines which meet these two directrices belong to $\Gamma$, so that $\Gamma$ may be described as follows: consider the directrices of the first kind of the points on an asymptotic curve on one focal sheet, and the corresponding directrices of the first kind along the corresponding asymptotic curve on the other focal sheet; then the linear complex to which the first asymptotic curve belongs is made up of the totality of lines which meet a pair of corresponding directrices. Evidently the corresponding asymptotic curve on the other sheet also belongs to the same linear complex.

Michigan Agricultural, Collegge, EAST LANSing, Mich. 